\title{
Magnetic interactions in iron superconductors studied with a five-orbital model within the Hartree-Fock and Heisenberg approximations
}

\author{
M.J. Calderón, G. León, B. Valenzuela, and E. Bascones* \\ Instituto de Ciencia de Materiales de Madrid, ICMM-CSIC, Cantoblanco, E-28049 Madrid (Spain).
}

(Dated: September 1, 2018)

\begin{abstract}
We have analyzed the magnetic interactions of a five orbital model for iron superconductors treated both within Hartree-Fock and Heisenberg approximations. We have found that the exchange constants depend non-trivially on the Fe-As-Fe angle and on the charge and orbital filling. Within the localized picture, columnar ordering is found for intermediate Hund's coupling $J_{H}$. At smaller $J_{H}$, an unusual orbital reorganization stabilizes checkerboard ordering. Ferromagnetism appears at large $J_{H}$. Ferromagnetic correlations are enhanced with electron doping while large hole doping stabilizes checkerboard antiferromagnetism, explaining the change in magnetic interactions upon substitution of $\mathrm{Fe}$ by $\mathrm{Co}$ or $\mathrm{Mn}$. For intermediate and large values of $U$, Hartree-Fock shows similar results as strong coupling though with a double stripe phase instead of ferromagnetism. Itinerancy enhances the stability of the columnar ordering. Comparison of the two approaches reveals a metallic region of the phase diagram where strong coupling physics is determinant.
\end{abstract}

PACS numbers: 75.10.Jm, 75.10.Lp, 75.30.Ds

\section{INTRODUCTION}

The origin of magnetism in iron superconductors, believed to be key to explain their high- $\mathrm{T}_{c}$ superconductivity, is still unsettled. Most iron pnictides order with $(\pi, 0)$ momentum, antiferromagnetically (AF) in the $x$-direction and ferromagnetically (FM) in the $y$ direction $\stackrel{1,2}{=}$ The itinerant versus strong coupling origin of magnetism is at the heart of the debate, with some authors proposing the coexistence of localized and itinerant moments. $\stackrel{-3-5}{-\underline{5}}$ In the itinerant picture the electrons close to the Fermi surface drive the ordering through approximate nesting $\underline{\underline{6}-\underline{9}}$ In the strong coupling limit localized spins interact AF. Classically, a $(\pi, 0)$ state arises when $J_{2}>J_{1} / 2$, with $J_{1}$ and $J_{2}$ the first and second nearest neighbor exchange parameters. A large $J_{2}$ was justified by the As-mediated exchange between Fe atoms. These, apparently opposite, views of magnetism are minimal descriptions of a more complex problem which includes the kinetic energy, the orbital character of the electronic bands, and the interactions between the electrons. In iron pnictides interactions are believed to be intermediate between both limits. 10

The applicability of strong coupling relies on $J_{2}>$ $J_{1} / 2$. However, little is known about the value of the exchange constants. Estimates for $J_{1}$ and $J_{2}$ have been restricted to a few ab-initio calculations for specific compounds,$\stackrel{11-15}{\underline{1}}$ and the extraction of the exchange parameters from neutron experiments is still controversial $16-18$ The relevance of longer range interactions has also been discussed $\stackrel{19}{\underline{19}}$

The situation is even more complex in FeTe which orders FM along one of the diagonals and AF along the other, in a double stripe (DS) pattern. No nesting features compatible with this ordering have been observed ${ }^{20}$ A large exchange interaction to third nearest neighbors $J_{3}$ has to be introduced to explain the stability of this

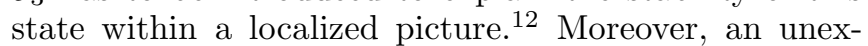

pected FM $J_{1}$ has been recently proposed to fit the spinwave spectrum of iron chalcogenides $\frac{18}{\underline{18}}$ Finally, the importance of Hund's coupling has also been emphasized in the literature $11,21-23$ though it is not clear at present how Hund's coupling affects magnetism.

In order to connect the itinerant and localized pictures, we here analyze the magnetic interactions of iron superconductors on the basis of a five orbital model ${ }^{24}$ treated both within HF and Heisenberg approximations. Within the strong coupling picture, we find that $J_{1}$ and $J_{2}$ have a non-trivial dependence on the atomic configuration and the Fe-As-Fe angle, and may become ferromagnetic at large Hund's coupling $J_{H}$. For undoped compounds, intermediate values of $J_{H}$ stabilize the $(\pi, 0)$ state, and the generally assumed relation $J_{2}>J_{1} / 2$ is fulfilled. $(\pi, \pi)$ checkerboard, with $J_{2}<J_{1} / 2$, is found for low $J_{H}$, while FM appears for high $J_{H}$. The checkerboard ordering at small $J_{H}$ is stabilized by an unusual orbital reorganization and results in an unexpected sensitivity of the ground state to crystal field parameters. The tendency towards FM, due to virtual transitions involving filled $3 z^{2}-r^{2}$ or $x^{2}-y^{2}$ orbitals, is enhanced with electron doping, while hole doping stabilizes checkerboard AF. This can explain the different magnetic orderings observed in the checkerboard antiferromagnetic $\mathrm{BaMn}_{2} \mathrm{As}_{2}, \stackrel{25}{=}$ with 5 electrons per $\mathrm{Mn}$, and the ferromagnetic LaOCoAs,$\stackrel{26}{2}$ with 7 electrons per Co. The HF description reproduces the $(\pi, \pi)-(\pi, 0)$ transition with increasing $J_{H}$ for intraorbital interaction $U \gtrsim 2.2 \mathrm{eV}$ allowing us to identify the metallic regions of the phase diagram where strong coupling physics is relevant. A DS state shows up at large $U$ and $J_{H}$, suggesting that it appears as a compromise between kinetic energy cost and the FM tendencies of localized spins. 

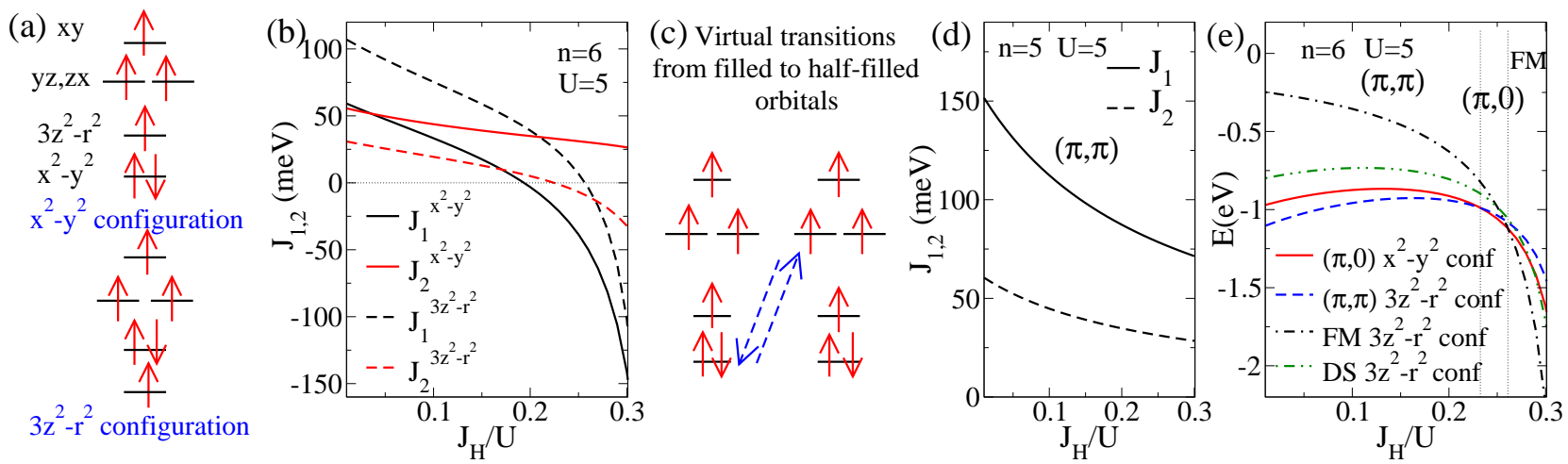

FIG. 1: (Color online) (a) Sketch of the two different orbital configurations $\left(\{\lambda\}=x^{2}-y^{2}\right.$ and $\left.\{\lambda\}=3 z^{2}-r^{2}\right)$ of the $S=2$ state for $n=6$. (b) Calculated exchange constants for $n=6, U=5 \mathrm{eV}$, and $\alpha=35.3^{\circ}$ as a function of Hund's coupling. (c) Illustration of the virtual transitions involving filled orbitals responsible for the FM exchanges for $n=6$ at large $J_{H} / U$. (d) Exchange constants as a function of $J_{H} / U$ for $n=5$ and $U=5 \mathrm{eV}$ assuming a $S=5 / 2$ state. A $(\pi, \pi)$ ground state is always favored. (e) Energies of the magnetic ground states as a function of $J_{H} / U$ for the same parameters as in (b). The double stripe DS order, although never the ground state, is included for comparison. Vertical lines separate regions with different ground states $[(\pi, \pi),(\pi, 0)$ and $\mathrm{FM}]$.

\section{MODEL}

The Hamiltonian includes intraorbital $U$, interorbital $U^{\prime}$, Hund's coupling $J_{H}$, and pair hopping $J^{\prime}$ terms.

$$
\begin{aligned}
& H=\sum_{i, j, \gamma, \beta, \sigma} t_{i, j}^{\gamma, \beta} c_{i, \gamma, \sigma}^{\dagger} c_{j, \beta, \sigma}+\text { h.c. }+U \sum_{j, \gamma} n_{j, \gamma, \uparrow} n_{j, \gamma, \downarrow} \\
& +\left(U^{\prime}-\frac{J_{H}}{2}\right) \sum_{j, \gamma>\beta, \sigma, \tilde{\sigma}} n_{j, \gamma, \sigma} n_{j, \beta, \tilde{\sigma}}-2 J_{H} \sum_{j, \gamma>\beta} \vec{S}_{j, \gamma} \vec{S}_{j, \beta} \\
& +J^{\prime} \sum_{j, \gamma \neq \beta} c_{j, \gamma, \uparrow}^{\dagger} c_{j, \gamma, \downarrow}^{\dagger} c_{j, \beta, \downarrow} c_{j, \beta, \uparrow}+\sum_{j, \gamma, \sigma} \epsilon_{\gamma} n_{j, \gamma, \sigma} .
\end{aligned}
$$

$i, j$ label the Fe sites in the Fe unit cell, $\sigma$ the spin and $\gamma$ and $\beta$ the five Fe d-orbitals $y z, z x, x y, 3 z^{2}-r^{2}$ and $x^{2}-y^{2}$, with $x$ and $y$ axis along the Fe-Fe bonds. We

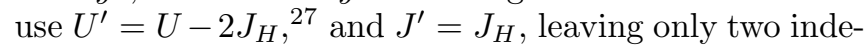
pendent interaction parameters, $U$ and $J_{H}$. The hopping amplitudes, restricted to first and second neighbors, depend on the angle $\alpha$ formed by the Fe-As bonds and the Fe-plane $\stackrel{24}{\underline{2}} \alpha=35.3^{\circ}$, corresponding to the regular Fe-As tetrahedra, is assumed except when indicated. We take ${ }^{24} \epsilon_{x y}=0.02, \epsilon_{z x, y z}=0, \epsilon_{3 z^{2}-r^{2}}=-0.55$ and $\epsilon_{x^{2}-y^{2}}=-0.6$ for the crystal field. Energies are in units of $(p d \sigma)^{2} /\left|\epsilon_{d}-\epsilon_{p}\right| \sim 1 \mathrm{eV}$, with $p d \sigma$ the $\sigma$ overlap between the Fe-d and As-p orbitals and $\left|\epsilon_{d}-\epsilon_{p}\right|$ their energy difference. ${ }^{24}$ For details on the mapping to a classical Heisenberg model and HF treatment see Appendix $\mathrm{A}$

To second order in perturbation theory, starting from localized atomic states, the 5-orbital Hamiltonian is mapped onto a classical Heisenberg Hamiltonian:

$$
E_{0}^{\{\lambda\}}+\frac{J_{1}^{\{\lambda\}}}{|S|^{2}} \sum_{\langle i, j\rangle} \vec{S}_{i} \vec{S}_{j}+\frac{J_{2}^{\{\lambda\}}}{|S|^{2}} \sum_{\langle\langle i, j\rangle\rangle} \vec{S}_{i} \vec{S}_{j}
$$

with $\vec{S}_{i}=\sum_{\beta} \vec{S}_{i, \beta}$ the atomic moment and $\langle i, j\rangle$ and $\langle\langle i, j\rangle\rangle$ restricted to first and second nearest neighbors respectively. Note that a bicuadratic term $\sim K\left(S_{i} S_{j}\right)^{2}$ has been discussed phenomenologically in connection with the structural transition, nematicity, and to reproduce the neutron spectra $\frac{17,18,28,29}{1}$ The prefactor $K$ would appear to higher order in $t / U$ in perturbation theory and is beyond the scope of this work. Similarly, longer range interactions $\frac{19}{9}$ are neglected.

To calculate the exchange constants $J_{1,2}^{\{\lambda\}}$ we focus on the largest possible spin state, with each orbital being half-filled or filled. For $n=6$, as in undoped compounds, this corresponds to $S=2$. This large spin state dominates the $(\pi, 0)$ mean field phase diagram at large $U, \underline{30,31}$ Due to the small crystal field splitting, we consider two possible atomic configurations, labelled by $\{\lambda\}$, for the $S=2$ state with filled $x^{2}-y^{2}$ or $3 z^{2}-r^{2}$ orbitals, see Fig. 10 (a).

\section{RESULTS}

As shown in Fig. 1(b) $J_{1}^{\{\lambda\}}$ and $J_{2}^{\{\lambda\}}$ decrease monotonically with Hund's coupling. Decreasing exchange constants with $J_{H}$ are generally expected, but the decrease we find is notably steep, mainly for $J_{1}$ and at large $J_{H} / U$, where a change in the slope happens and the exchange constants even become FM. This strong dependence at large $J_{H}$ is due to virtual transitions from a filled orbital to a half-filled one on a neighbor atom, see Fig. 1(c) and Appendix $\mathrm{A}$. These transitions are favored by the small crystal field splitting characteristic of iron pnictides.

The exchange constants and their $J_{1} / J_{2}$ ratio are very different in both atomic configurations. For $n=6$, in the $x^{2}-y^{2}$ configuration favored by the crystal field splitting, $J_{2}^{x^{2}-y^{2}}>J_{1}^{x^{2}-y^{2}} / 2$ and the $(\pi, 0)$ state is lowest in energy, except at large $J_{H} / U$ for which FM is expected. When comparing the energies between the ordered states in both orbital configurations in Fig. 1(e), the $(\pi, \pi)$ or- 
der with filled $3 z^{2}-r^{2}$ becomes the ground state in a wide range of parameters. The selection of $(\pi, \pi)$ in the $3 z^{2}-r^{2}$ configuration is in accordance with the exchange constants ratio $J_{2}^{3 z^{2}-r^{2}}<J_{1}^{3 z^{2}-r^{2}} / 2$. Remarkably, the gain in magnetic energy, associated to the large value of the direct hopping $t_{x^{2}-y^{2}, x^{2}-y^{2}} \stackrel{24}{\stackrel{2}{\leftrightarrows}}$ compensates for the cost in crystal field. Due to the small energy difference between the two states, very small changes in the crystal field can stabilize $(\pi, 0)$ for smaller values of $J_{H}$, see Appendix B

Note that the exchange constants in the $(\pi, 0)$ and $(\pi, \pi)$ states may be different. Thus estimating these constants by comparing the energy of the different magnetic states with those predicted by a mapping to a Heisenberg model ${ }^{12-15}$ with the same $J_{1}$ and $J_{2}$ may lead to errors. Moreover, our results indicate that the filled orbitals are not inert for magnetism, so the use of 4-orbital models which neglect them is questionable ${ }^{21.32}$

With electron doping, the number of filled orbitals increases. For $n=7,(\pi, 0)$ order is found at small $J_{H}$ and FM behavior appears at a smaller value of $J_{H}$ compared to $n=6$ (not shown). On the other hand, there are no filled orbitals at $n=5$ in its highest spin state ( $S=5 / 2$ ), implying a weaker dependence on $J_{H} / U$ of the exchange parameters, always AF, see Fig. 1(d). In this case $J_{2}<J_{1} / 2$ and $(\pi, \pi)$ ordering is found for all $J_{H}$. Therefore, a clear asymmetry is found in the magnetic interactions with strong electron-hole doping. This is consistent with experimental observations: $\mathrm{BaMn}_{2} \mathrm{As}_{2}$ and LaOCoAs show checkerboard ordering and ferromagnetism, respectively $\underline{25,26}$

A $(\pi, \pi)-(\pi, 0)$ transition with increasing $J_{H}$ is also present in the HF phase diagram in Fig. 2. Crystal field sensitivity and orbital reorganization, similar to that found in the localized picture, is realized, see Appendix $\mathrm{B}$. The transition between $(\pi, \pi)$ and $(\pi, 0)$ is accompanied by a charge transfer between $x^{2}-y^{2}$ and $3 z^{2}-r^{2}$. In the $(\pi, 0)$ state, $3 z^{2}-r^{2}$ is emptied while $x^{2}-y^{2}$ gets filled as $U$ increases $\frac{30}{2}$ In the $(\pi, \pi)$ state, $3 z^{2}-r^{2}$ fills with increasing $U$ while the other orbitals tend to half filling. The $(\pi, 0)$ state becomes more stable if the splitting between $x^{2}-y^{2}$ and $3 z^{2}-r^{2}$ increases, see Appendix B.

As previously found, $\stackrel{30}{=}$ two different $(\pi, 0)$ states show up at the HF level. In the LM state, opposite orbital magnetizations within the same atom result in a low magnetic moment which violates Hund's rule. This state is stabilized thanks to the anisotropy of the orbital exchange constants ${ }^{30}$ and has been proposed to explain the low magnetic moment found experimentally $30,33,34$ In the HM state, all the orbital magnetizations point in the same direction. The strong coupling predictions, dashed lines in Fig. 2, are valid for comparison with this HM state.

As expected within a weak coupling description, and opposite to the strong coupling predictions, for small values of the interaction a HM $(\pi, 0)$ is found close to the non-magnetic boundary. In this region nesting seems to

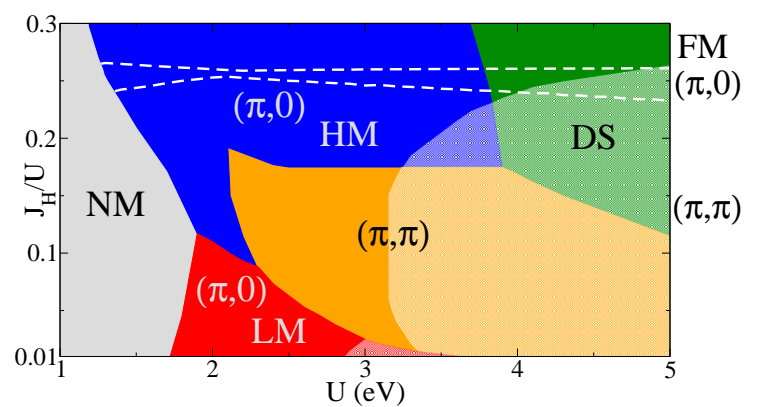

FIG. 2: (Color online) Hartree Fock magnetic phase diagram calculated at fixed density $n=6$ as a function of $U$ and $J_{H} / U$. Grey stands for non-magnetic (NM), blue for the $\operatorname{AF}(\pi, 0)$ high moment (HM) state satisfying Hund's rule, red for a the AF $(\pi, 0)$ low moment (LM) state which violates Hund's rule, orange for $\operatorname{AF}(\pi, \pi)$ and green for the double stripe (DS) state with charge modulation (see text). Shaded areas mark insulating phases. Superposed dashed lines on the magnetic regions show the phase transition lines between the $(\pi, \pi)$ and $\mathrm{HM}(\pi, 0)$ states and between the HM $(\pi, 0)$ and the FM phase predicted by the $S=2$ Heisenberg model.

stabilize the $(\pi, 0)$ ordering, although the electronic reconstruction happens not only at the Fermi surface but also at higher energies. Similar physics has been discussed within density functional theory calculations ${ }^{23}$ On the other hand, the stability of the $(\pi, \pi)$ state for $U \gtrsim 2.2 \mathrm{eV}$, the orbital reorganization at the transition, and the strong crystal field sensitivity cannot be understood within the nesting picture and are a clear signature of localized physics. Note that this is found even for metallic states. Even for $U \gtrsim 2.2 \mathrm{eV}$ the $(\pi, 0)$ state is more prominent in the HF phase diagram than in the Heisenberg description. This suggests some influence of itinerancy in stabilizing the $(\pi, 0)$ state.

At large $J_{H}$ the kinetic energy effects present at the HF level prevent the FM solution found in the strong coupling description to arise. Instead, a double stripe solution is found at large $U$ and $J_{H}$. This DS state is charge modulated ( 5 or 7 electrons for the $n=6$ case) in a checkerboard fashion. Note that in the Heisenberg picture, a homogeneous DS state, though not the ground state, is lower in energy than both $(\pi, \pi)$ and $(\pi, 0)$ at large $J_{H} / U$, see Fig. 1(e). The homogeneous DS state, with $n$ electrons in each atom, is difficult to stabilize in the HF calculations, mainly at large $J_{H}$. When stabilized, it has larger energy than the charge modulated DS state. As shown in Fig. 3, the DS state is unstable towards phase separation at $n=6$, see Appendix $\mathrm{A}$, but can be stabilized by electron doping. As expected for first order transitions, PS appears at the boundary between the different magnetic phases. The instability at $n \gtrsim 6.5$, which only involves the DS state, has a different origin: it is caused by a negative compressiblity related to the charge modulation.

Fig. 3(b) also evidences an electron-hole asymmetry around the undoped composition $n=6$. In agreement with the strong coupling predictions, for $n=5(\pi, \pi)$ 

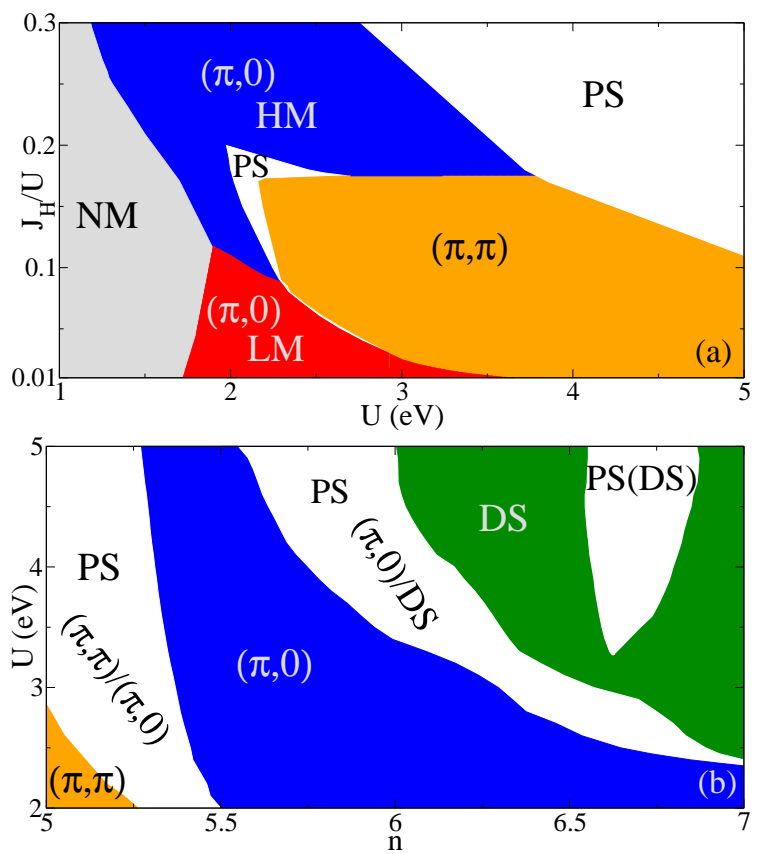

FIG. 3: (Color online) Mean field magnetic phase diagram in the grand canonical ensemble as a function of $U$ and $J_{H} / U$ and for $n=6$ (a) and as a function of $U$ and $n$ for $J_{H} / U=$ 0.22 (b). Same color code as in Fig. (2) applies. White regions are unstable towards phase separation.

correlations dominate, even at large $J_{H}$ (calculations at $J_{H} / U=0.28$ give similar results). When $n$ increases, the $(\pi, 0)$ state becomes lower in energy, being the ground state in a wide region of parameters. For $n>6$ and intermediate or large $U$ the DS state is found. A larger tendency to DS solutions with increasing $n$ is also expected in the Heisenberg description. Within HF, the electron-hole asymmetry close to $n=6$ gets reduced as $U$ decreases.

We finally analyze the effect of the Fe-As-Fe geometry on the magnetic interactions. A direct relation between the Fe-As-Fe angle and the critical superconducting temperature $^{2,35,36}$ as well as on the magnetic ordering ${ }^{18}$ has been claimed. As illustrated in Fig. 4, the exchange constants vary non-monotonically with $\alpha$ due to the relative importance of the hopping (to both first and second neighbors) via the As $\stackrel{24,37}{2}$ The dependences of $J_{1}$ and $J_{2}$ on $\alpha$ are different for the $x^{2}-y^{2}$ and $3 z^{2}-r^{2}$ configurations, with $J_{1} / J_{2}$ changing with $\alpha$. Checkerboard and ferromagnetic ordering are more stable for elongated tetrahedra.

\section{SUMMARY}

We have analyzed the magnetic interactions and ground state of a five orbital model for iron pnictides by means of Heisenberg and Hartree Fock approaches. We have calculated the exchange constants of the strong coupling model and show that their value and sign depend

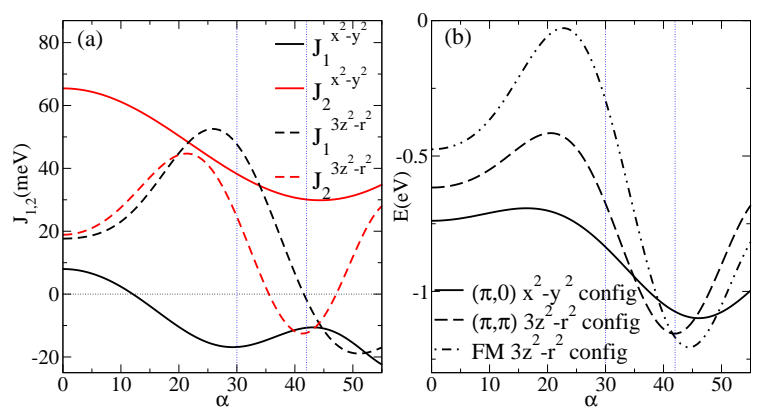

FIG. 4: (Color online) (a) First and second nearest neighbor exchange constants $J_{1}^{\{\lambda\}}$ and $J_{2}^{\{\lambda\}}$ as a function of $\alpha$, the angle formed by the Fe-As bond and the Fe plane, for $n=6, U=5$ $\mathrm{eV}$, and $J_{H} / U=0.22$. (b) Energies of the most competitive magnetic orderings as a function of $\alpha$ for the parameters in (a). The sequence of magnetic orderings with increasing $\alpha$ is $(\pi, 0),(\pi, \pi)$, and FM. Vertical lines delimitate the experimentally relevant values of $\alpha$.

non-trivially on the Fe-As-Fe angle, the orbital filling, the number of electrons per Fe, and the Hund's coupling $J_{H}$. A $(\pi, \pi)-(\pi, 0)$ transition which involves orbital reorganization is present in both approaches. This cannot be explained within the nesting picture and allows us to identify a metallic region of the phase diagram where strong coupling physics is relevant. On the contrary the magnetic ordering close to the non-magnetic transition could be due to nesting. Both pictures seem to be important to describe magnetism in iron pnictides. Our results uncover the effect of $J_{H}$ on the stabilization of $(\pi, 0)$ ordering. Due to virtual transitions involving filled orbitals, a large $J_{H}$ produces a strong decrease of the exchange constants leading to small AF or even FM $J_{1}$. This could help understand the neutron scattering ex-

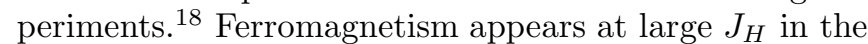
Heisenberg approach, while a double stripe phase shows up in Hartree-Fock. Electron doping enhances ferromagnetic (double-stripe) tendencies, while large hole-doping leads to checkerboard ordering, in agreement with experiments $\stackrel{25,26}{2}$

Note added. Recently, similar results with hole doping were obtained by the variational Monte Carlo method, 38

We have benefited from conversations with F. Guinea, A. Chubukov and N. Perkins. We acknowledge funding from Ministerio de Ciencia e Innovación through Grants No. FIS 2008-00124, FIS 2009-08744 and Ramón y Cajal contract, and from CSIC through Grants No. PIE2009601033 and PIE-200960I180.

\section{Appendix A: Calculation details}

Starting from localized atomic states we map the 5orbital interacting Hamiltonian of Eq.(1) in a classical Heisenberg Hamiltonian with exchange interactions to first and second nearest neighbors 


$$
E_{0}^{\{\lambda\}}+\frac{J_{1}^{\{\lambda\}}}{|S|^{2}} \sum_{\langle i, j\rangle} \vec{S}_{i} \vec{S}_{j}+\frac{J_{2}^{\{\lambda\}}}{|S|^{2}} \sum_{\langle\langle i, j\rangle\rangle} \vec{S}_{i} \vec{S}_{j} .
$$

Here $\{\lambda\}$ labels the considered orbital configuration (as defined in Fig. 1 (a)), $\vec{S}_{i}=\sum_{\beta} \vec{S}_{i, \beta}$ the atomic moment and $\langle i, j\rangle$ and $\langle\langle i, j\rangle\rangle$ are restricted to first and second nearest neighbors respectively. We restrict to atomic states with maximum total spin $S(S=2$ for $n=6$ and $S=5 / 2$ for $n=5)$ and maximum $\left|S_{z}\right|\left(S_{z}= \pm 2\right.$ and $S_{z}= \pm 5 / 2$ for $n=6$ and $n=5$ respectively). With this, $E_{0}^{\{\lambda\}}$ and $J_{1,2}^{\{\lambda\}}$ are

$$
\begin{gathered}
E_{0}^{\{\lambda\}}=\sum_{\langle i, j\rangle,\langle\langle i, j\rangle\rangle} \frac{E_{P ; i, j}^{\{\lambda\}}+E_{A P ; i, j}^{\{\lambda\}}}{4}, \\
J_{1,2}^{\{\lambda\}}=\frac{E_{P ; i, j}^{\{\lambda\}}-E_{A P ; i, j}^{\{\lambda\}}}{4},
\end{gathered}
$$

with $i, j$ two first (second) nearest neighbors for $J_{1}\left(J_{2}\right)$. $E_{P ; i, j}^{\{\lambda\}}$ and $E_{A P ; i, j}^{\{\lambda\}}$ are the energies corresponding to two parallel or antiparallel spins at $i$ and $j$. The factor of 4 (instead of 2) corrects the bond double-counting in Eq.(A1). When using Eqs. (A2) and (A3) we are neglecting quantum fluctuations. In this sense we are treating the spin classically. As discussed in the text and below, the classical approximation provides good understanding on the magnetic interactions dominant in iron pnictides. Quantum fluctuations are expected to be of little importante for large spins. The spin states $S=2$ and $S=5 / 2$ discussed in the text are large enough to justify the classical treatment of spins.

$\mathrm{P}$ and $\mathrm{AP}$ energies can be written in terms of atomic and magnetic energies

$$
E_{P, A P ; i, j}^{\{\lambda\}}=E_{a t}^{\{\lambda\}}+E_{P, A P ; i, j}^{\{\lambda\}, \operatorname{mag}},
$$

where $E_{a t}^{\{\lambda\}}$ accounts for the interaction and crystal field energy to charge the atoms to the selected $n=6$ or $n=5$ states, while $E_{P, A P ; i, j}^{\{\lambda\}, \operatorname{mag}}$ is the magnetic contribution. $E_{a t}^{\{\lambda\}}$ enters in the value of the constant $E_{0}^{\{\lambda\}}$ but cancels out in the expression of the exchange constants $J_{1,2}^{\{\lambda\}}$. We simplify $E_{a t}^{\{\lambda\}}$ to $E_{a t}^{x^{2}-y^{2}}=0$ and $E_{a t}^{3 z^{2}-r^{2}}=\epsilon_{3 z^{2}-r^{2}-}$ $\epsilon_{x^{2}-y^{2}}$ per atom, as the other terms drop out from the calculation. For $n=6$

$$
\begin{array}{r}
E_{A P ; i, j}^{\{\lambda\}, m a g}=-\sum_{\eta, \nu} \frac{2\left(t_{i, j}^{\nu, \eta}\right)^{2}}{J^{\prime 2}+\Delta E_{-}^{2}}\left(\frac{\Delta E_{-}^{2}}{U+3 J_{H}+\epsilon_{\eta}-\epsilon_{\nu}+\Delta E_{+}}\right. \\
\left.+\frac{J^{\prime 2}}{U+3 J_{H}+\epsilon_{\eta}-\epsilon_{\nu}+\Delta E_{-}}\right) \\
-\sum_{\nu} 2\left(t_{i, j}^{\lambda, \nu}\right)^{2}\left(\frac{1 / 5}{U-3 J_{H}+\epsilon_{\eta}-\epsilon_{\lambda}}+\frac{4 / 5}{U+2 J_{H}+\epsilon_{\eta}-\epsilon_{\lambda}}\right)
\end{array}
$$

Here $\eta$ and $\nu$ label half-filled orbitals and $\lambda$ refers to the filled one; $t_{i, j}^{\beta, \gamma}$, are the hopping amplitudes between orbitals $\beta$ and $\gamma$ in atoms $i$ and $j$ respectively $\underline{24} \Delta E_{ \pm}=$ $\epsilon_{\nu}-\epsilon_{\lambda} \pm\left(\left(\epsilon_{\lambda}-\epsilon_{\nu}\right)^{2}+J^{\prime 2}\right)^{1 / 2}$. The first term in Eq. (A55) includes virtual transition from half-filled orbitals in one atom to half-filled orbitals in a neighbour atom and takes into account that the intermediate state is not an eigenstate of the pair hopping operator. The second term is associated with virtual transitions of an electron from the filled orbital to a half-filled orbital in the other atom. Only the electron whose spin is opposite to the magnetic moment of the neighbor atom can hop. The state left behind is not an eigenstate of the Hund's term.

When the magnetic moments of the two atoms are parallel the only transitions allowed involve the filled orbital. The contribution of these transitions is

$$
E_{P ; i, j}^{\{\lambda\}, \operatorname{mag}}=-\sum_{\nu} \frac{2\left(t_{i, j}^{\lambda, \nu}\right)^{2}}{U-3 J_{H}+\epsilon_{\lambda}-\epsilon_{\nu}} .
$$

For $n=5$ and $S=5 / 2, E_{P ; i, j}^{S=5 / 2, m a g}=0$ and

$$
E_{A P ; i, j}^{S=5 / 2, m a g}=-\sum_{\nu \eta} \frac{2\left(t_{i, j}^{\eta, \nu}\right)^{2}}{U+4 J_{H}+\epsilon_{\eta}-\epsilon_{\nu}} .
$$

In this latter case, the exchange constants are clearly AF. Whether a $(\pi, \pi)$ or $(\pi, 0)$ state is favoured depends on the relative values of $J_{1}$ and $J_{2}$. As shown in Fig. 1, for $n=5(\pi, \pi)$ ordering is always preferred.

The $n=6$ case is more interesting. For $J_{H}=0$ the contribution of the transitions which depart from filled orbitals is the same with $\mathrm{P}$ or AP ordering. Consequently they do not affect the magnetic ordering, and the exchange constants, driven by the transitions from half-filled orbitals are AF. With increasing $J_{H}$ their contribution is larger in the $\mathrm{P}$ case. At a given $J_{H}$ these transitions favor FM ordering. For small $J_{H}$ the transitions from half-filled orbitals still dominate, but at large $J_{H} J_{1}^{\{\lambda\}}$ and $J_{2}^{\{\lambda\}}$ become ferromagnetic. Due to different $t_{i, j}^{\gamma, \beta}$ in each of these terms the value of $J_{H}$ at which this happens is different for each exchange constant.

Another interesting aspect regards $E_{0}^{\{\lambda\}}$. The contribution of $E_{P, A F ; i, j}^{\{\lambda\}, \operatorname{mag}}$ to $E_{0}^{\{\lambda\}}$ can be seen as the energy gain associated to creating magnetic moments, even if on average they do not order. When mapping to Heisenberg models it is usual to disregard $E_{0}$ because if a single atomic state is selected its value does not affect the differences in energy between magnetic states. However, for $n=6$, the small crystal field splitting between $x^{2}-y^{2}$ and $3 z^{2}-r^{2}$ requires the inclusion of two different atomic states. Due to different hopping amplitudes involving these orbitals, $E_{0}^{x^{2}-y^{2}} \neq E_{0}^{3 z^{2}-r^{2}}$ is found. This can be observed in Fig. 5 as a function of the Fe-As-Fe and Hund's coupling. Consequently $E_{0}^{\{\lambda\}}$ helps select the atomic state $\{\lambda\}$, with the a priori unexpected result that the atomic state in which $3 z^{2}-r^{2}$ is filled is 

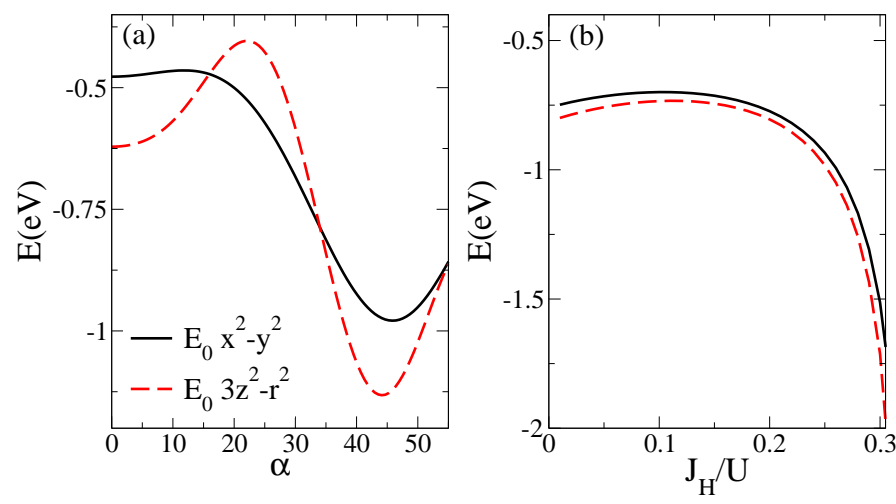

FIG. 5: (Color online) Dependence of $E_{0}^{x^{2}-y^{2}}$ and $E_{0}^{3 z^{2}-r^{2}}$ for $n=6$ and $U=5 \mathrm{eV}$ as a function of the Fe-As-Fe angle $\alpha$ for $J_{H} / U=0.22$ (a) and Hund's coupling for $\alpha=35.3^{\circ}(\mathrm{b})$.

preferred in a wide range of parameters. Note that the formation of magnetic moments is favoured in the experimentally relevant range of $\mathrm{Fe}-\mathrm{As}-\mathrm{Fe}$ angle and at large Hund's coupling. The large values of $E_{0}^{\{\lambda\}}$ also explain the large values of the total energy, despite the relatively small values of the exchange constants.

We have compared these predictions with a selfconsistent mean-field Hartree Fock calculation which includes non-magnetic (NM), FM, and AF states with $Q=(\pi, 0), Q=(\pi, \pi)$, and DS ordering. In the DS calculation the system is divided into two interpenetrating lattices coupled via first nearest neighbor hopping terms. The axis and orbital basis is rotated and a $(\pi, 0)$ state along the Fe-diagonals is assumed. In the mean-field calculation only spin and orbital-diagonal average terms are kept:

$$
\begin{aligned}
n_{\gamma} & =\sum_{k, \sigma}\left\langle c_{k, \gamma, \sigma}^{\dagger} c_{k, \gamma, \sigma}\right\rangle \\
m_{\gamma} & =\sum_{k}\left[\left\langle c_{k+\mathbf{Q}, \gamma, \uparrow}^{\dagger} c_{k, \gamma, \uparrow}\right\rangle-\left\langle c_{k+\mathbf{Q}, \gamma, \downarrow}^{\dagger} c_{k, \gamma, \downarrow}\right\rangle\right] .
\end{aligned}
$$

We have checked that this approximation does not have any influence on the mean field results: Disregarded terms are of order $\sim 10^{-4}$. Several initial configurations for $n_{\gamma}$ and $m_{\gamma}$ are probed for each ordered state. A self-consistent solution is considered to be ordered (insulating) when the magnetic moment $m$ (gap) is larger than 0.001 .

The phase diagrams in Fig. 2 and Fig. 7 (right panel) have been obtained comparing the energies of the meanfield solutions at fixed density. Different phases have a different energy $E$ versus density $n$ relation. Jumps in the chemical potential $\mu$ appear at first-order boundaries between ordered phases. In such situations phase separation is expected. In a range of densities the system decreases its energy by allowing relative fractions of the two neighboring magnetic phases. $n=6$ may belong to this range of densities for values of the interactions
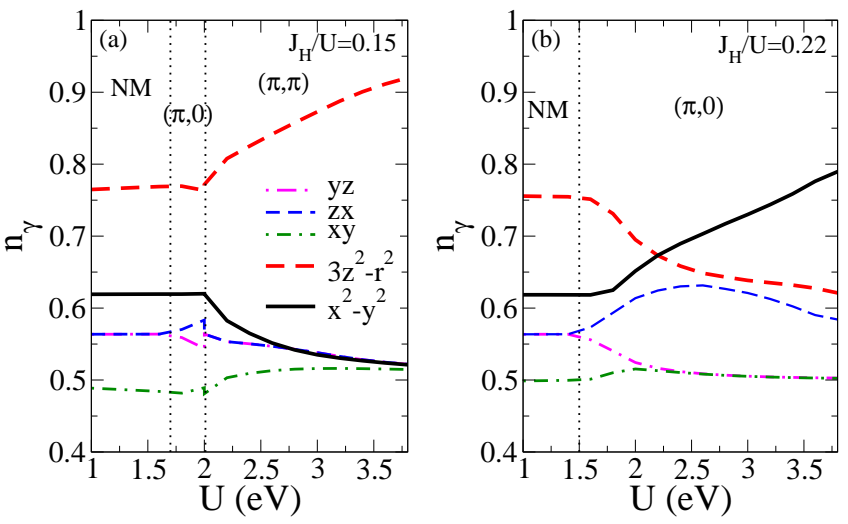

FIG. 6: (Color online) (a) and (b) Dependence of the orbital filling as a function of the intraorbital interaction for $J_{H} / U=$ 0.15 and $J_{H} / U=0.22$ respectively. Vertical lines separate regions with different ground states.

close to phase boundaries. To determine the region of phase space unstable towards phase separation in Fig. 3 we work in the grand-canonical ensemble (fixed chemical potential) and compare $E-\mu n$ for the different states. The chemical potential can be calculated as $\partial E / \partial n$ or by looking at the energy of the last state occupied in metallic systems, both methods giving the same value. $\mu$ jumps discontinuously when crossing a gap. According to Maxwell's construction, phase boundaries between two phases 1 and 2 are given by $E_{1}-\mu n_{1}=E_{2}-\mu n_{2}$. Neither phase 1 nor phase 2 are stable in the range $n_{1}<n<n_{2}$. A phase separated mixture of both phases arises.

Phase separation for a given phase will also appear with negative compressibility, i.e. $\mu$ decreases with increasing $n$. This is observed in the DS state in several ranges of $n$, including $n=6$ and $n \leq 6.5$. In this case phase separation happens between states with the same magnetic ordering but different density. Densities $n_{1}$ and $n_{2}$ are determined as above. Note, that while at $n=6$ DS is intrinsically unstable towards phase separation, at this density phase separation between DS and $(\pi, 0)$ is favored.

\section{Appendix B: $(\pi, 0)$ versus $(\pi, \pi)$. Crystal field sensitivity}

Fig. 2 shows the phase diagram corresponding to $n=6$ calculated at fixed density. The mean field ground state evolves from a non-magnetic solution with zero magnetic moment and orbital fillings close to the non-interacting ones at small $U$ to $(\pi, 0)$ and $(\pi, \pi)$ states with $m=4 \mu_{B}$, with $\mu_{B}$ a Bohr magneton, as in an atomic $S=2$ state at large $U$. As seen in Fig. 6, in the $(\pi, 0)$ state every orbital except $x^{2}-y^{2}$ tends to half filling at large $U$, $x^{2}-y^{2}$ becoming completely filled. This orbital filling is 

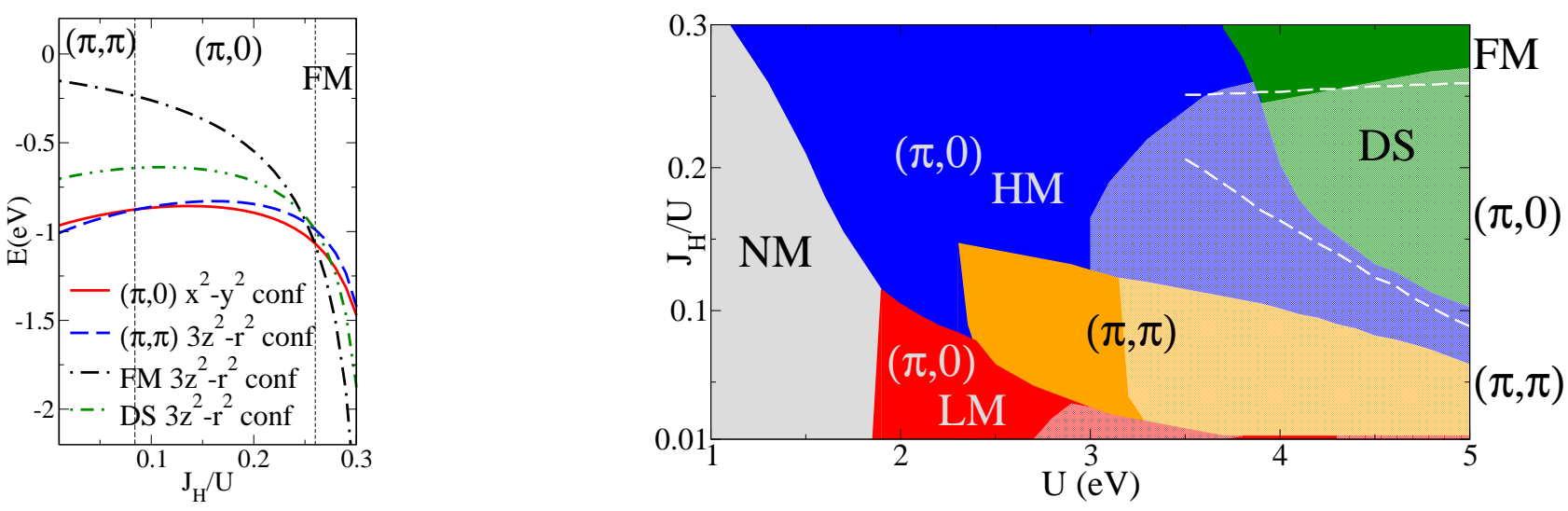

FIG. 7: (Color online) (Left) Energies of the magnetic ground states as a function of $J_{H} / U$ calculated within the Heisenberg picture and (right) Hartree-Fock phase diagram for the same parameters as in Fig. 2 and Fig. 3, except for the crystal field splitting $\epsilon_{3 z^{2}-r^{2}}-\epsilon_{x^{2}-y^{2}}$ which is $100 \mathrm{meV}$ larger here. In the right panel the color code is the same as in Fig. 2. Superposed dashed lines on the magnetic regions show the phase transition lines between the $(\pi, \pi)$ and HM $(\pi, 0)$ states and between the HM $(\pi, 0)$ and the FM phase predicted by the $S=2$ Heisenberg model.

expected on the basis of the crystal field splitting. On the other hand, in the $(\pi, \pi)$ state, it is the $3 z^{2}-r^{2}$ orbital the one which fills completely while $x^{2}-y^{2}$ tends to halffilling. This orbital filling competes with the crystal field, in agreement with the strong coupling predictions.

The competition between crystal field and magnetic energy suggests a strong sensitivity of the $(\pi, \pi)-(\pi, 0)$ transition to the crystal field splitting $\epsilon_{3 z^{2}-r^{2}}-\epsilon_{x^{2}-y^{2}}$. Such a sensitivity is manifest in Fig. 7 where the stability of the different phases is shown for a crystal field splitting $\epsilon_{3 z^{2}-r^{2}}-\epsilon_{x^{2}-y^{2}}=150 \mathrm{meV}, 100 \mathrm{meV}$ larger than the one used in Fig. 2 and Fig. 3. This modification of the crystal field is below the accuracy of the tight-binding. The region of stability of the $(\pi, 0)$ state in Fig. 7 is considerably larger than in Figs. 2 and 3.

This orbitally reorganized $(\pi, \pi)$ state is a clear signature of strong-coupling physics for $U \gtrsim 2.2 \mathrm{eV}$. On the contrary, its absence for smaller values of $U$ suggests a nesting-driven $(\pi, 0)$ state.
* Electronic address: leni@icmm.csic.es

${ }^{1}$ C. de la Cruz, Q. Huang, J. Lynn, J. Li, W. Ratcliff, J. Zarestky, H. Mook, G. Chen, J. Luo, N. Wang, et al., Nature 453, 899 (2008).

2 J. Zhao, Q. Huang, C. de la Cruz, S. Li, J. Lynn, Y. Chen, M. Green, G. Chen, G. Li, Z. Li, et al., Nature Materials 7, 953 (2008).

3 J. Wu, P. Phillips, and A. H. Castro Neto, Phys. Rev. Lett. 101, 126401 (2008).

${ }^{4}$ Q. Si, E. Abrahams, J. Dai, and J.-X. Zhu, New Journal of Physics 11, 045001 (2009).

5 W.-G. Yin, C.-C. Lee, and W. Ku, Physical Review Letters 105, 107004 (2010).

6 I. Mazin, M. D. Johannes, L. Boeri, and D. S. K. Koepernik, Phys. Rev. B 78, 085104 (2008).

7 S. Raghu, X. Qi, C.-X. Liu, D. Scalapino, and S.-C. Zhang, Phys. Rev. B 77, 220503 (2008).

8 A. Chubukov, D. Efremov, and I. Eremin, Phys. Rev. B 78, 134512 (2008).

${ }^{9}$ V. Cvetkovic and Z. Tesanovic, Europhysics Lett. 85, 37002 (2009).

10 M. Qazilbash, J. Hamlin, R. Baumbach, L. Zhang, D. Singh, M. Maple, and D. Basov, Electronic correlations in the iron pnictides 5, 647 (2009).

11 K. Haule and G. Kotliar, New Journal of Physics 11, 025021 (2009).
12 F. Ma, W. Ji, J. Hu, Z.-Y. Lu, and T. Xiang, Phys. Rev. Lett. 102, 177003 (2009).

13 C.-Y. Moon, S. Y. Park, and H. J. Choi, Phys. Rev. B 80, $054522(2009)$.

14 C.-Y. Moon and H. J. Choi, Phys. Rev. Lett. 104, 057003 (2010).

15 X.-W. Yan, M. Gao, Z.-Y. Lu, and T. Xiang, arXiv:1012.5536.

16 J. Zhao, D. T. Adroja, D.-X. Yao, R. Bewley, S. Li, X. F. Wang, G. Wu, X. H. Chen, J. Hu, and P. Dai, Nature Physics 5, 555 (2009).

17 A. Wysocki, K. Belashchenko, and V. P. Antropov, Nature Physics 7, 485 (2011).

18 J. Hu, B. Xu, W. Liu, N.-N. Hao, and Y. Wang, arXiv:1106.5169.

19 T. Yildirim, Physica C 469, 425 (2009).

${ }^{20}$ Y. Xia, D. Qian, L. Wray, D. Hsieh, G. F. Chen, J. L. Luo, N. L. Wang, and M. Z. Hasan, Phys. Rev. Lett. 103, 037002 (2009).

21 P. Hansmann, R. Arita, A. Toschi, S. Sakai, G. Sangiovanni, and K. Held, Phys. Rev. Lett. 104, 197002 (2010).

22 Z. P. Yin, K. Haule, and G. Kotliar, Nature Physics 7, 294 (2010).

23 M. Johannes and I. Mazin, Phys. Rev. B 79, 220510R (2009).

24 M. J. Calderón, B. Valenzuela, and E. Bascones, Phys. 
Rev. B 80, 094531 (2009).

${ }^{25}$ Y. Singh, M. Green, Q. Huang, A. Kreyssig, R. McQueeney, D. Johnston, and A. Goldman, Phys. Rev. B 80, 100403(R) (2009).

${ }^{26}$ H. Yanagi, R. Kawamura, T. Kamiya, Y. Kamihara, M. Hirano, T. Nakamura, H. Osawa, and H. Hosono, Physical Review B 77, 224431 (2008).

27 C. Castellani, C. R. Natoli, and J. Ranninger, Phys. Rev. B 18, 4945 (1978).

28 C. Xu, M. Müller, and S. Sachdev, Phys. Rev. B 78, 020501 (2008).

29 C. Fang, H. Yao, W.-F. Tsai, J. Hu, and S. A. Kivelson, Phys. Rev. B 77, 224509 (2008).

30 E. Bascones, M. J. Calderón, and B. Valenzuela, Phys. Rev. Lett. 104, 227201 (2010).

31 B. Valenzuela, E. Bascones, and M. J. Calderón, Phys.
Rev. Lett. 105, 207202 (2010).

32 M. Laad and L. Craco, arxiv:1010.2940.

33 F. Cricchio, O. Granas, and L. Nordstrom, Phys. Rev. B 81, 140403 (2009).

${ }^{34}$ G.-Q. Liu, arXiv:1105.5412v1 (2011).

35 C. H. Lee, A. Iyo, H. Eisaki, H. Kito, M. T. FernandezDiaz, T. Ito, K. Kihou, H. Matsuhata, M. Braden, and K. Yamada, J. Phys. Soc. Jpn. 77, 083704 (2008).

${ }^{36}$ K. Kuroki, H. Usui, S. Onari, R. Arita, and H. Aoki, Phys. Rev. B 79, 224511 (2009).

37 The effect of the Fe-As-Fe angle on the crystal field has been neglected.

38 T. Misawa, K. Nakamura, and M. Imada, Phys. Rev. Lett. 108, 177007 (2012). 